\title{
Experimental and theoretical study of lattice relaxation around refractory atoms in nickel
}

\author{
V. Koteski ${ }^{\text {a,c, }, *}$, H.-E. Mahnke ${ }^{\mathrm{a}, \mathrm{b}}$, J. Belošević-Čavor ${ }^{\mathrm{c}}$, B. Cekićc ${ }^{\mathrm{c}}$, G. Schumacher ${ }^{\mathrm{a}}$ \\ ${ }^{a}$ Hahn-Meitner-Institut Berlin GmbH, Bereich SF, Glienicker Strasse 100, D-14109 Berlin, Germany \\ b Freie Universität Berlin, Fachbereich Physik, Arnimallee 14, D-14195 Berlin, Germany \\ ${ }^{\mathrm{c}}$ Institute of Nuclear Sciences Vinca, P.O. Box 522, 11001 Belgrade, Serbia
}

Received 26 February 2008; received in revised form 8 May 2008; accepted 13 May 2008

Available online 19 June 2008

\begin{abstract}
The lattice relaxation around $\mathrm{Mo}, \mathrm{Ru}, \mathrm{Hf}, \mathrm{Ta}, \mathrm{W}$ and $\mathrm{Re}$ in $\mathrm{Ni}$ is investigated by means of X-ray absorption spectroscopy. For all of the investigated concentrations the substitutional lattice position is confirmed except for the higher Hf concentration where different phases are observed. An outward relaxation of the neighboring atoms is detected with clear trends of increasing nearest neighbor distances with decreasing valence for the $5 \mathrm{~d}$ impurities, and separate trends for the $4 \mathrm{~d}$ impurities. Ab initio supercell calculations within the linearized augmented plane wave formalism are used to complement the experimental results and allow for a better interpretation of the experimental trends in terms of the electronic structure of the impurity.

(c) 2008 Acta Materialia Inc. Published by Elsevier Ltd. All rights reserved.
\end{abstract}

Keywords: Extended X-ray absorption fine structure (EXAFS, XANES); Nickel alloys; Augmented plane wave method (FLAPW)

\section{Introduction}

While in many important applications of metals and intermetallic phases the properties of materials can be tailored by introducing suitable alloying elements, the local structure and possible lattice relaxation around these elements is in general not very well known. Nickel-base superalloys are of significant technological importance, due to their very high strength (shear resistance and flow stress) at high temperatures, as compared to usual alloys such as steels. The last generations of superalloys include refractory elements, e.g. Mo, W, Ru, or Re, that help enhance their superior mechanical properties and corrosion resistance [1]. The strengthening effect is not fully understood in terms of the underlying mechanism, and efforts have been made to correlate it to structural, electronic or bonding features of the impurity system [2-4].

\footnotetext{
* Corresponding author. Address: Institute of Nuclear Sciences Vinca, P.O. Box 522, 11001 Belgrade, Serbia.

E-mail address: vkotes@vin.bg.ac.yu (V. Koteski).
}

Ni-base superalloys consist of a face-centred cubic (fcc) Ni solid solution matrix ( $\gamma$ phase) in which the strengthening $\gamma^{\prime}$ phase $\left(\mathrm{Ni}_{3} \mathrm{Al}\right.$-type) is dispersed. The lattice mismatch between the two phases is found to be an important parameter in controlling the properties of the superalloys [5-7]. It appears that one way of altering the lattice mismatch is by doping with refractory elements, which incorporated either into the $\gamma$ or $\gamma^{\prime}$ phase, or into both, can contribute to the overall contraction or expansion of the corresponding lattice. The clustering of the refractory elements has also been attributed as one of the possible mechanisms of solid solution strengthening [2].

In the following we present the results of a combined, experimental and theoretical determination of the local structure around $\mathrm{Mo}, \mathrm{Ru}, \mathrm{Hf}, \mathrm{Ta}, \mathrm{W}$ and $\mathrm{Re}$ in nickel. In some model $\gamma$ phases of Ni-base superalloys the addition of small amounts of Re, Mo, Ru and $\mathrm{W}$ were shown to affect short-range order (SRO) which is reflected by peaks of the $\{11 / 20\}$ type. The SRO is approximately stable up to $600{ }^{\circ} \mathrm{C}$ and then decreases gradually up to about $1000{ }^{\circ} \mathrm{C}$ where it finally vanishes $[8,9]$. To date, the lattice 